\title{
35. RESULTS OF X-RAY-MINERALOGY ANALYSES OF SAMPLES FROM DEEP SEA DRILLING PROJECT SITES 415 AND 416, MOROCCAN BASIN
}

\author{
CEPM Laboratory, Elf Aquitaine, Pau, France
}

\section{INTRODUCTION}

$\mathrm{X}$-ray-diffraction analyses of 164 samples were conducted on whole-rock samples (for determination of gross mineralogy) and on the carbonate-free fractions (for determination of the clay-mineral content). The analyses were carried out in the laboratory of the Société Nationale Elf-Aquitaine (Production) in Pau, France. Results are given in Tables 1 and 2 and in Figures 1, 2, and 3.

\section{ANALYTICAL METHODS}

Determinations of quartz, calcite, and dolomite contents were made from analysis of isogranular powder
$(40 \mathrm{~mm})$ of whole rock. The corresponding X-ray diffractograms were processed by computer and rendered as per cent weight of the whole rock.

Determinations of clay-mineral contents were made by analysis of the carbonate-free fraction of $5 \mathrm{~mm}$ or less. Kaolinite, illite, chlorite, attapulgite, and sepiolite contents were estimated in relative percentages from the heights and areas of the X-ray diffraction peaks. OpalCT is also estimated in relative percentages, but the presence of clinoptilolite is only indicated by the notation " 1. ."

TABLE 1

X-Ray-Mineralogy Data for Site 415

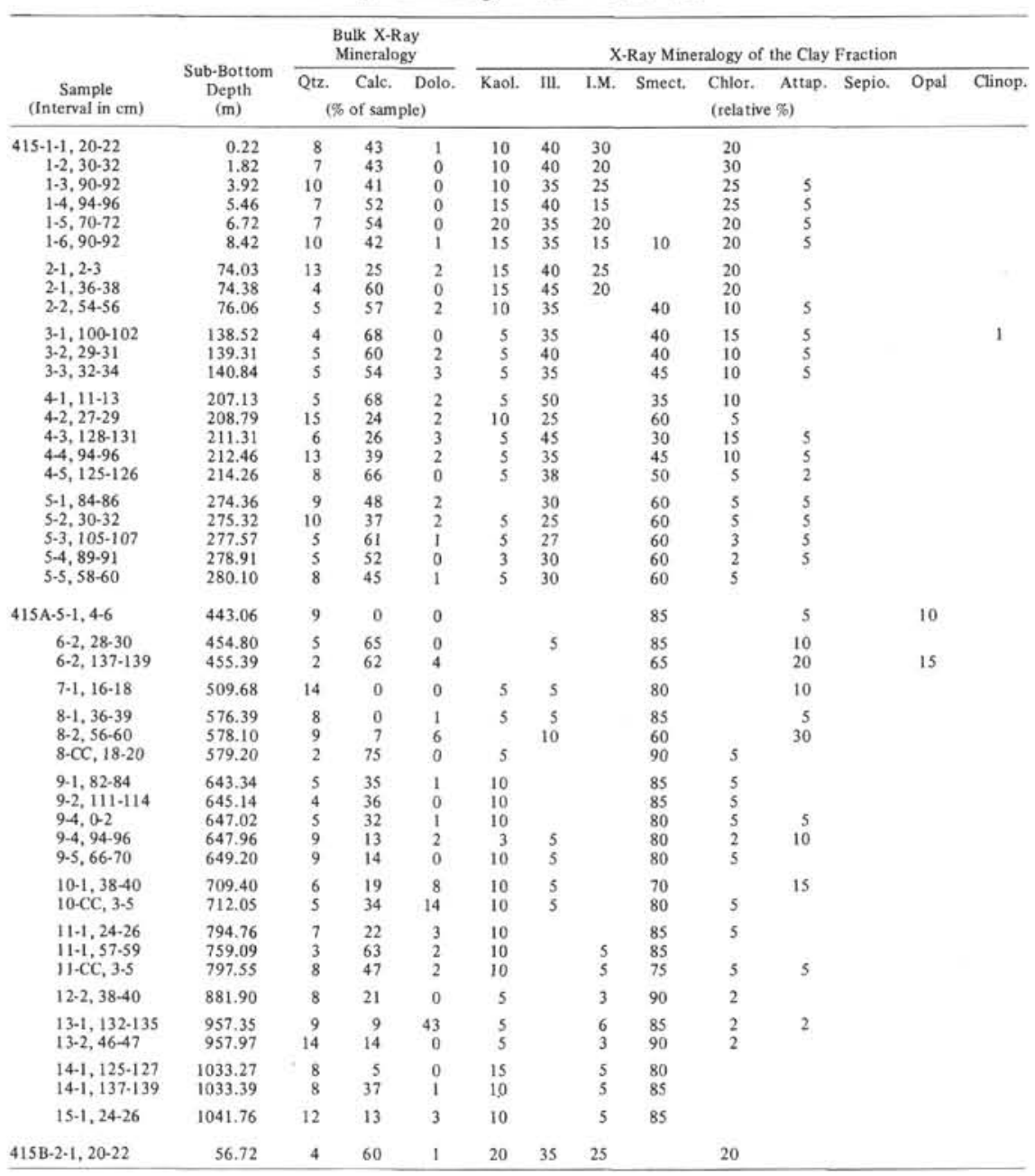


TABLE 2

X-Ray-Mineralogy Data for Site 416

\begin{tabular}{|c|c|c|c|c|c|c|c|c|c|c|c|c|c|}
\hline \multirow[b]{2}{*}{$\begin{array}{c}\text { Sample } \\
\text { (Interval in } \mathrm{cm} \text { ) }\end{array}$} & \multirow[b]{2}{*}{$\begin{array}{l}\text { Sub-Bottom } \\
\text { Depth } \\
\text { (m) }\end{array}$} & \multicolumn{3}{|c|}{$\begin{array}{l}\text { Bulk X-Ray } \\
\text { Mineralogy }\end{array}$} & \multicolumn{9}{|c|}{ X-Ray Mineralogy of the Clay Fraction } \\
\hline & & \multicolumn{3}{|c|}{$\begin{array}{l}\text { Qtz. Calc. Dolo. } \\
(\% \text { of sample })\end{array}$} & \multirow{2}{*}{$\begin{array}{c}\text { Kaol. } \\
10\end{array}$} & \multirow{2}{*}{$\begin{array}{l}\text { III. } \\
40\end{array}$} & \multirow{2}{*}{ I.M. } & \multirow{2}{*}{$\begin{array}{c}\text { Smect. } \\
35\end{array}$} & \multirow{2}{*}{$\begin{array}{l}\begin{array}{l}\text { Chlor. } \\
\text { (rela tive }\end{array} \\
15\end{array}$} & $\begin{array}{l}\text { Attap. } \\
\%)\end{array}$ & \multirow[t]{2}{*}{ Sepio. } & \multirow[t]{2}{*}{ Opal } & Clinop. \\
\hline $416 \mathrm{~A}-1-1,11-15$ & 146.15 & 13 & 38 & 0 & & & & & & & & & \\
\hline $1-1,20-21$ & 146.21 & 14 & 40 & 2 & 10 & 45 & 10 & 10 & 20 & 5 & & & \\
\hline $1-1,51-52$ & 146.52 & 5 & 61 & 1 & 10 & 40 & 20 & & 25 & 5 & & & \\
\hline $1-1,62-64$ & 146.64 & 4 & 69 & 0 & 10 & 55 & 10 & & 25 & & & & \\
\hline $1-1,91-93$ & 146.93 & 10 & 37 & 8 & 5 & 50 & & 25 & 15 & 5 & & & \\
\hline $\begin{array}{l}2-1,78-80 \\
2-1,86-87\end{array}$ & $\begin{array}{l}298.80 \\
298.87\end{array}$ & $\begin{array}{l}11 \\
13\end{array}$ & $\begin{array}{l}28 \\
16\end{array}$ & $\begin{array}{l}4 \\
4\end{array}$ & $\begin{array}{l}5 \\
5\end{array}$ & $\begin{array}{l}25 \\
40\end{array}$ & 20 & 35 & $\begin{array}{l}10 \\
10\end{array}$ & $\begin{array}{l}25 \\
25\end{array}$ & & & \\
\hline $2-2,25-27$ & 299.77 & 10 & 36 & 5 & 10 & 35 & & 35 & 15 & 5 & & & \\
\hline $2-2,32-34$ & 299.84 & 22 & 19 & 6 & 10 & 30 & & 35 & 5 & 20 & & & \\
\hline $2-4,30-32$ & 312.82 & 11 & 30 & 8 & & 35 & & 25 & 10 & 30 & & & \\
\hline $2-4,34-36$ & 312.86 & 12 & 20 & 7 & & 35 & & 25 & 10 & 30 & & & \\
\hline $3-3,8-10$ & 453.10 & 21 & 0 & 4 & 5 & 25 & & 55 & 5 & 10 & & & \\
\hline $3-3,15-17$ & 453.17 & 17 & 14 & 6 & 5 & 30 & & 40 & 5 & 20 & & & \\
\hline $3-3,94-97$ & 453.97 & 18 & 14 & 3 & & 35 & & 40 & 5 & 10 & & & \\
\hline $5-1,8-11$ & 754.11 & 6 & 0 & 14 & & & & & & 45 & 55 & & \\
\hline $6-1,30-32$ & 891.32 & 24 & 0 & 0 & 15 & 60 & 20 & & 5 & & & & \\
\hline $6-1,37-39$ & 891.39 & 22 & 0 & 0 & 10 & 60 & 25 & & 5 & & & & \\
\hline $6-3,24-26$ & 894.26 & 38 & 0 & 0 & 15 & 55 & 20 & & 10 & & & & \\
\hline $6-3,146-148$ & 895.48 & 25 & 0 & 0 & 10 & 55 & 20 & & 5 & 10 & & & \\
\hline $7-1,61-63$ & 982.63 & 25 & 3 & 0 & 20 & 45 & 25 & & 10 & & & & \\
\hline $7-3,117-118$ & 986.18 & 12 & 10 & 0 & 25 & 35 & & 30 & 10 & & & & \\
\hline $9-2,8-9$ & 1179.04 & 26 & 17 & 0 & 20 & 40 & & 35 & 5 & & & & \\
\hline $9-2,11-12$ & 1179.07 & 31 & 21 & 0 & 20 & 40 & 30 & & 5 & 5 & & & \\
\hline $9-2,20-22$ & 1179.17 & 41 & 19 & 0 & 20 & 25 & 20 & 20 & 10 & 5 & & & \\
\hline $9-4,40-41$ & 1181.91 & 14 & 15 & 0 & 15 & 35 & 10 & 20 & 15 & 5 & & & \\
\hline $9-4,45-46$ & 1181.96 & 43 & 12 & 0 & 20 & 30 & 10 & 30 & 10 & 5 & & & \\
\hline $10-1,48-51$ & 1185.51 & 15 & 10 & 0 & 30 & 35 & 10 & 20 & & 5 & & & 1 \\
\hline $11-2,40-41$ & 1196.91 & 12 & 15 & $\begin{array}{l}0 \\
0\end{array}$ & $\begin{array}{l}20 \\
20\end{array}$ & 40 & 10 & 25 & $\begin{array}{l}5 \\
5\end{array}$ & & & & \\
\hline $\begin{array}{l}11-2,49-52 \\
11-2,63-65\end{array}$ & $\begin{array}{l}1197.02 \\
1197.15\end{array}$ & $\begin{array}{l}14 \\
30\end{array}$ & $\begin{array}{r}4 \\
12\end{array}$ & 0 & $\begin{array}{l}20 \\
15\end{array}$ & $\begin{array}{l}40 \\
40\end{array}$ & $\begin{array}{l}10 \\
10\end{array}$ & $\begin{array}{l}25 \\
25\end{array}$ & $\begin{array}{r}5 \\
10\end{array}$ & & & & \\
\hline $12-1,15-18$ & 1204.18 & 11 & 5 & 0 & 10 & 10 & & 75 & 5 & & & & \\
\hline $12-4,48-50$ & 1209.00 & 9 & 39 & 0 & 15 & 35 & 10 & 30 & 5 & 5 & & & \\
\hline $12-4,68-71$ & 1209.21 & 42 & 14 & 1 & 20 & 35 & 15 & 20 & 5 & 5 & & & \\
\hline $13-2,43-47$ & 1214.97 & 16 & 1 & 0 & 25 & 40 & 10 & 15 & 5 & 5 & & & \\
\hline $13-2,57-59$ & 1215.09 & 9 & 13 & 0 & 30 & 40 & 20 & & 5 & 5 & & & \\
\hline $14-2,42-44$ & 1223.94 & 11 & 31 & 0 & 30 & 40 & 20 & & 5 & 5 & & & \\
\hline $14-2,60-62$ & 1224.12 & 15 & 2 & 0 & 15 & 40 & 30 & & 15 & & & & \\
\hline $14-2,65-66$ & 1224.16 & 41 & 7 & 0 & 20 & 35 & 20 & & 20 & 5 & & & \\
\hline $16-1,84-86$ & 1243.86 & 28 & 0 & 0 & 15 & 35 & & 35 & 10 & 5 & & & \\
\hline $16-1,129-131$ & 1244.31 & 8 & 45 & 0 & 20 & 40 & & 30 & 10 & 5 & & & \\
\hline $16-1,143-144$ & 1244.44 & 34 & 22 & 2 & 25 & 35 & 30 & & 10 & & & & \\
\hline $17-2,65-67$ & 1254.17 & 15 & 0 & 0 & 15 & 50 & 20 & & 15 & & & & \\
\hline $17-2,127-129$ & 1254.71 & 10 & 29 & 0 & 25 & 40 & 30 & & 5 & & & & \\
\hline $19-3,5-7$ & 1274.07 & 13 & 22 & 0 & 20 & 45 & 20 & 10 & 5 & & & & \\
\hline $19-3,13-15$ & 1274.15 & 13 & 0 & 0 & 20 & 35 & 10 & 25 & 5 & 5 & & & \\
\hline $19-3,34-36$ & 1274.36 & 44 & 2 & 0 & 15 & 35 & 10 & 25 & 15 & & & & \\
\hline $19-3,46-48$ & 1274.48 & 13 & 9 & 0 & 20 & 35 & 25 & & 15 & 5 & & & \\
\hline $19-3,49-51$ & 1274.51 & 43 & 8 & 0 & 15 & 30 & 10 & 25 & 15 & 5 & & & \\
\hline $21-1,72-74$ & 1290.74 & 15 & 2 & 0 & 15 & 30 & 40 & & 10 & 5 & & & \\
\hline $21-1,106-108$ & 1291.08 & 12 & 6 & 0 & 25 & 35 & 30 & & 10 & & & & \\
\hline $21-3,82-84$ & 1293.84 & 9 & 38 & 0 & 25 & 35 & 20 & 10 & 5 & 5 & & & \\
\hline $21-3,127-129$ & 1294.29 & 47 & 6 & 0 & 15 & 35 & & 35 & 15 & & & & \\
\hline $21-5,108-111$ & 1297.11 & 9 & 36 & 0 & 20 & 40 & & 35 & 5 & & & & \\
\hline $22-1,124-125$ & 1300.25 & 32 & 9 & 0 & 10 & 40 & & 40 & 10 & & & & \\
\hline $22-1,134-136$ & 1300.36 & 9 & 48 & 0 & 20 & 35 & 10 & 30 & 5 & & & & \\
\hline $22-1,140-142$ & 1300.42 & 12 & 18 & 0 & 15 & 35 & 25 & 10 & 10 & 5 & & & \\
\hline $22-2,14-16$ & 1300.66 & 14 & 5 & 0 & 15 & 40 & 20 & 15 & 10 & & & & \\
\hline $22-4,125-127$ & 1304.77 & 11 & 12 & 0 & 20 & 40 & 20 & & 15 & 5 & & & \\
\hline
\end{tabular}


TABLE 2 - Continued

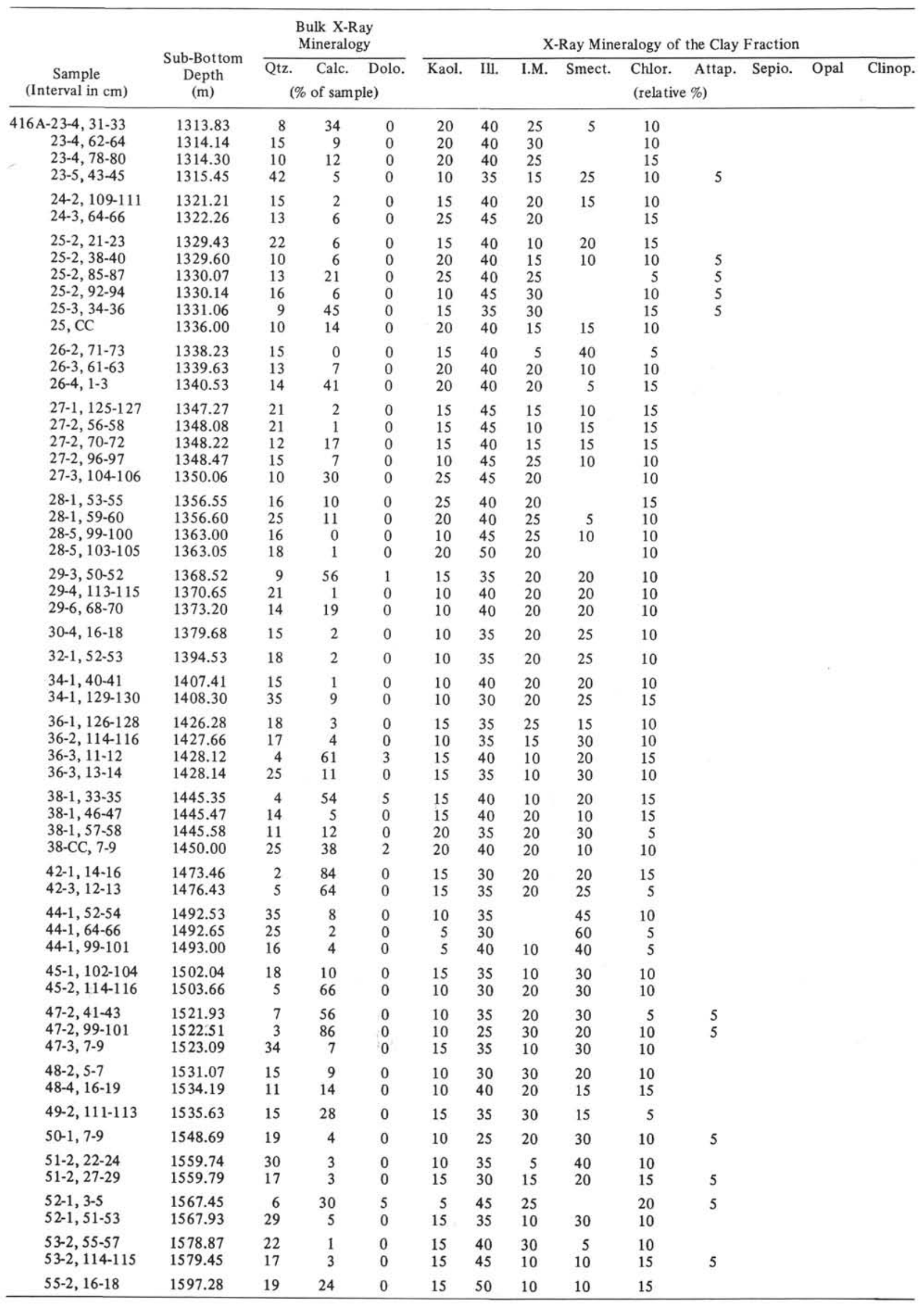




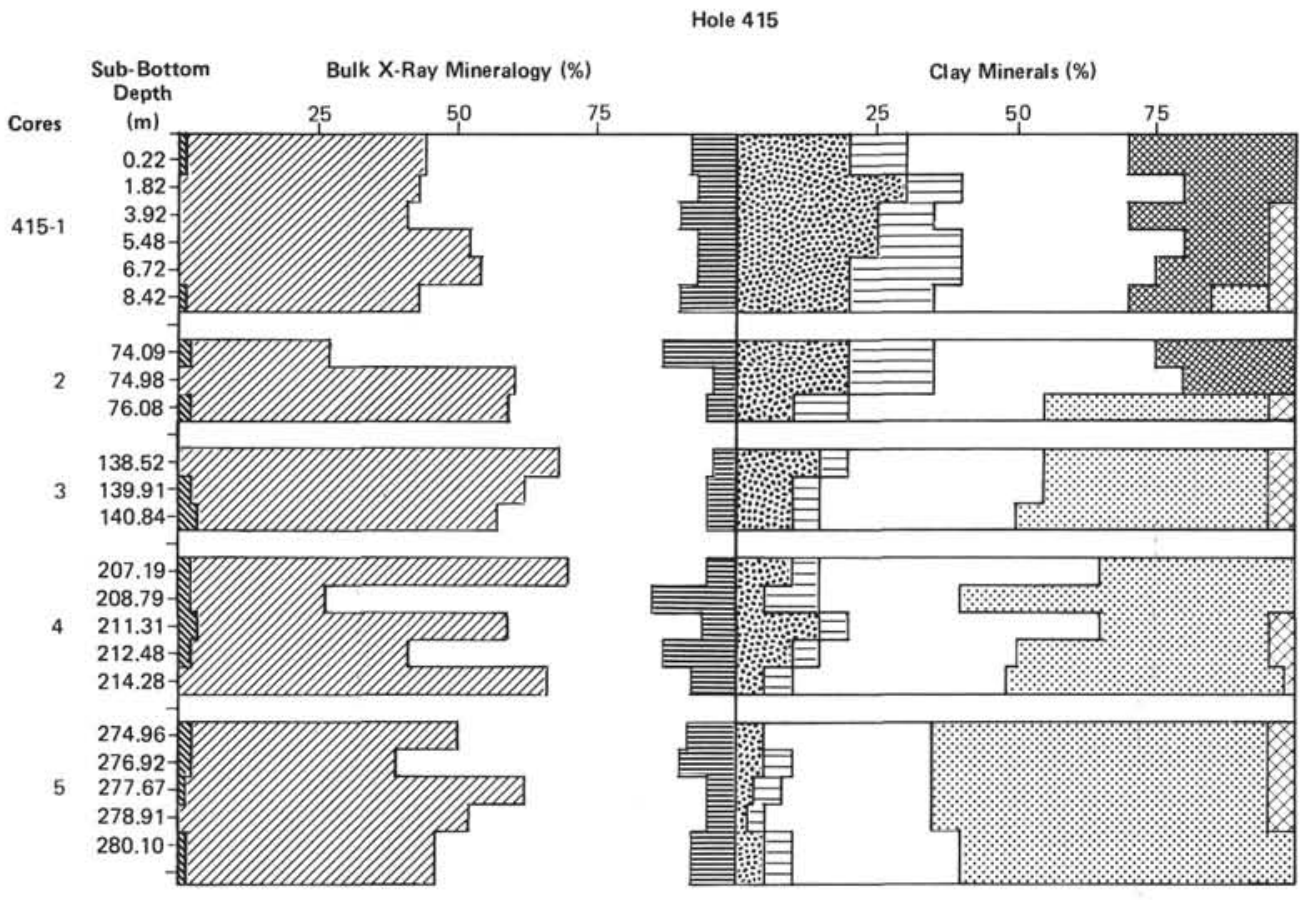

\begin{tabular}{|c|c|c|}
\hline \multicolumn{3}{|c|}{ Key } \\
\hline Dolomite & Chlorite & Smectite \\
\hline Calcite & Eaolinite & $\otimes$ Attapulgite \\
\hline Quartz & Illite & Illite-Smectite \\
\hline Opal-CT & Sepiolite & \\
\hline
\end{tabular}

Figure 1. Graphic plot of X-ray-mineralogy data for samples from Hole 415. 


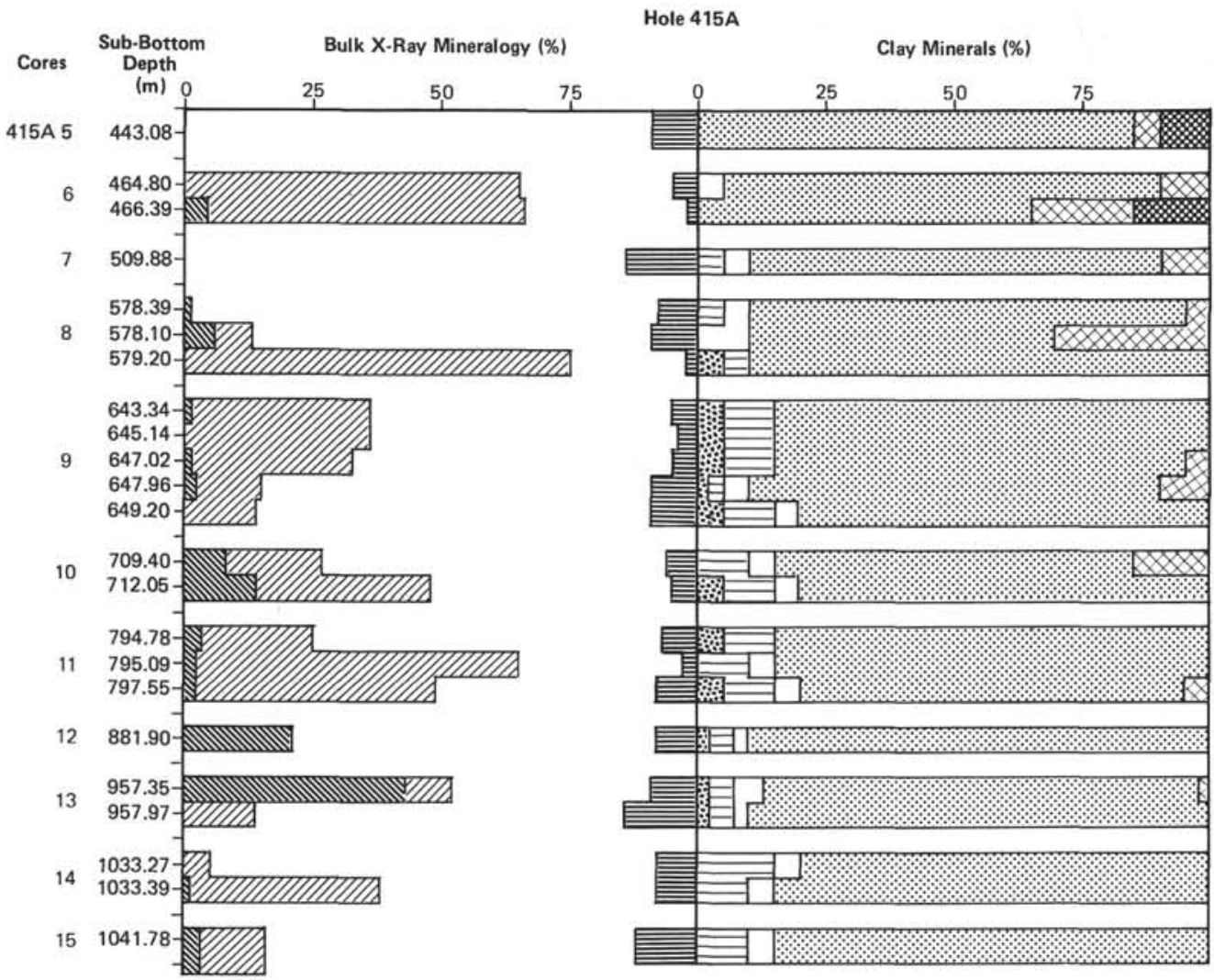

Figure 2. Graphic plot of X-ray-mineralogy data for samples from Hole 415A. Symbols as in Figure 1. 


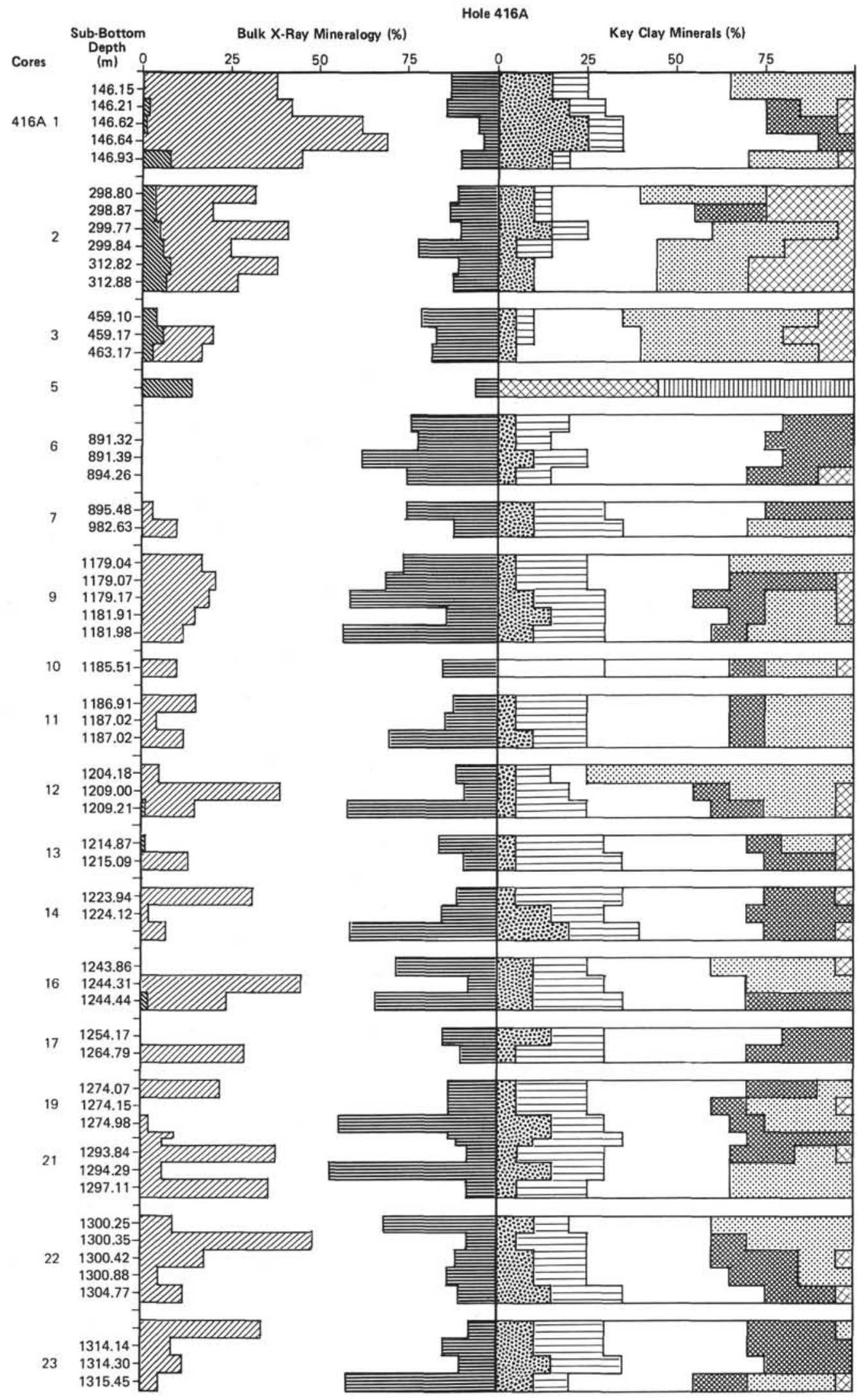

Figure 3. Graphic plot of X-ray-mineralogy data for samples from Hole 416A. Symbols as in Figure 1. 


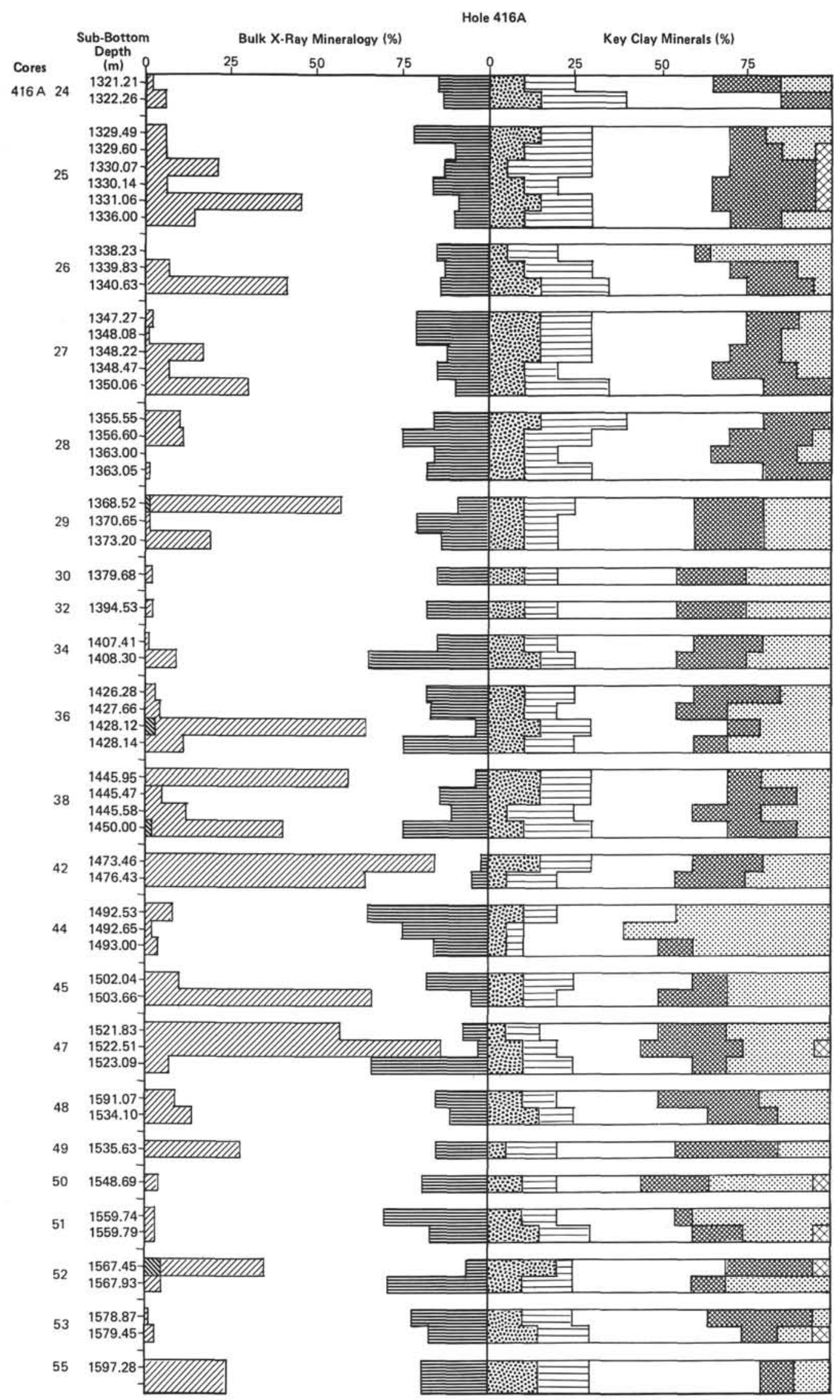

Figure 3. (Continued). 